Experiment and theory building

Author(s): Lydia Patton

Source: Synthese, Vol. 184, No. 3 (February 2012), pp. 235-246

Published by: Springer

Stable URL: https://www.jstor.org/stable/41411191

Accessed: 14-02-2019 13:46 UTC

JSTOR is a not-for-profit service that helps scholars, researchers, and students discover, use, and build upon a wide range of content in a trusted digital archive. We use information technology and tools to increase productivity and facilitate new forms of scholarship. For more information about JSTOR, please contact support@jstor.org.

Your use of the JSTOR archive indicates your acceptance of the Terms \& Conditions of Use, available at https://about.jstor.org/terms

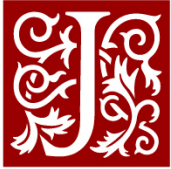




\title{
Experiment and theory building
}

\author{
Lydia Patton
}

Received: 21 March 2010 / Accepted: 4 August 2010/ Published online: 18 August 2010 (C) Springer Science+Business Media B.V. 2010

\begin{abstract}
I examine the role of inference from experiment in theory building. What are the options open to the scientific community when faced with an experimental result that appears to be in conflict with accepted theory? I distinguish, in Laudan's (1977), Nickels's (1981), and Franklin's (1993) sense, between the context of pursuit and the context of justification of a scientific theory. Making this distinction allows for a productive middle position between epistemic realism and constructivism. The decision to pursue a new or a revised theory in response to the new evidence may not be fully rationally determined. Nonetheless, it is possible to distinguish the question of whether there is reason to pursue a theory from the question of whether that theory, once it has been pursued over time, solves a problem of interest to science. I argue that, in this context, there is a solid way to distinguish between the contexts of pursuit and of justification, on the basis of a theory's evidential support and problem-solving ability.
\end{abstract}

Keywords Experiment - Theory building · Realism - Constructivism · Pursuit · Justification · Inference · Helmholtz · Fluid mechanics · Hacking · Franklin $\cdot$ Nickles $\cdot$ Problem solving

\section{Inference from experiment: constructivism and epistemic realism}

The question of the role of experiment in scientific inference has been gathering steam in the philosophy of science. This can be seen in the work of, e.g., Franklin (1986 and 1993), Franklin-Hall (2005), Hacking (1983), Galison (1987), Mayo (1996), and Nickles (1980). The question of how to justify inference from experiment, especially

\footnotetext{
L. Patton $(\otimes)$

Virginia Tech, Blacksburg, Virginia, USA

e-mail: critique@vt.edu
} 
inference with respect to the confirmation or disconfirmation of hypotheses, has been illuminated by this recent literature. The pragmatic element of inference from experiment, the question of calibration, of how experiments end-all are key questions in this arena. My topic of choice is not restricted to the evaluation of specific hypotheses, however. Instead, I examine the response of scientists to experiments that are in prima facie conflict with accepted theory.

I analyze the debate between epistemic realism and constructivism in giving a philosophical account of the relationship between experiment and theory. While the individual positions differ, the following analysis is intended as a statement of the general basis of disagreement between epistemic realists and constructivists. Epistemic realists claim that experiment can yield warrants, proofs, or justifications (though defeasible ones) for scientific claims, which are true or approximately true. Examples of such realists are Putnam (in his recent naïve realist incarnation), Peter Smith, and Alan Musgrave. Constructivists counter that the fit between experiment and theoretical claims is loose, and that the chain of justification for accepting a given claim must be filled in with "constructions" that could include, for instance, Kuhn's epistemic values or the politics of the scientific community. Examples of the kinds of constructivists I mean are Kuhn (of course), Fine, and van Fraassen-I am not limiting the discussion to social constructivism.

It seems that for every move one side can make, the other has a countermove. Epistemic realists give examples of experiments that ineluctably prove some result, whereupon constructivists respond that any given such experiment doesn't prove the result given distinct auxiliary hypotheses. Or, constructivists can appeal to Larry Laudan's pessimistic meta-induction, according to which all scientific theories to date have been falsified so we can infer that all current ones will as well, or to Harry Collins's experimenter's regress, according to which experiments can be adjusted to prove any scientific claim by revising the principles of inference involved or by fiddling with the instruments (cf. Laudan 1981; Collins 1994). In response, epistemic realists can appeal to Lakatos's explanation of when scientific change is warranted, in terms of whether a result or justified claim challenges the hard or the soft core of a theory.

But epistemic realists and constructivists should agree on one point of common ground. It is desirable for experiments to reveal novel effects, effects that drive the progress of science and test the scope of scientific claims. The practice of science is an enterprise of discovery as well as an engine of proof and certainty. What happens, then, when exploratory experiment reveals an effect that could not have been predicted a priori from known principles? This is a hard case for both sides, and it is the case I will examine.

In so doing, I will make several assumptions. The first is the Einsteinian view that scientific explanation progresses in two ways: by constructing pictures of complex phenomena out of more simple elements, and by testing the scope of scientific laws and principles and the values of physical constants in novel contexts. The second is that the right way to evaluate the relationship between scientific claims, theories, and observed effects is to combine the axiomatic method, of stating the laws, principles, and axioms of a theory and drawing conclusions from these deductively, with pragmatic linking rules between phenomenology and theory. This is the sense in which I will read "predicted a priori": whether a given observed effect can be represented as a deductive 
(including a deductive probabilistic) consequence of the law or principle at stake, plus the currently accepted pragmatic rules for linking phenomenology to theory.

The hard case I stated above, then, can be restated more specifically. What happens when a novel effect is observed in experiment that cannot be derived a priori from known theory plus phenomenology? This may not seem a hard case for an epistemic realist. After all, every scientific law we have has a collection of observed facts to support it. However, the situation under analysis presents a need for new principles or laws, or at least for revisions to the existing ones, and thus supports the pessimistic meta-induction. For instance, recently an exception to Planck's law of black body radiation has been observed. ${ }^{\prime}$ If this result is confirmed, the epistemic realist must make a choice. Has Planck's law been defeated or falsified, or has it been limited in scope? If the latter is the case, what is the status of the truth of the theory the epistemic realist sees as warranted? If a theory is accepted as true, what does it mean to say that the scope of that theory can be limited or revised?

Things appear little better in the constructivist camp. An exception to a law has been found. It is incumbent on scientists to determine how to integrate this result into the theory involved. The scientific community decides what will take place: will the theory be patched up to take the aberrant result into account? Will a new theory be constructed? Will the community instead discard the result by arguing from the experimenter's regress, that properly functioning equipment would not have yielded this result? The constructivist's strength is in explaining these phenomena after the fact. After the scientific community has decided how to move on, constructivists are very good at reconstructing their decisions. But constructivists tend to demur, or to question the basis of the question itself, when asked (1) whether what the scientists have done was the right choice or constitutes progress, or (2) to predict what they will do. This is another way of characterizing the debate: epistemic realists are in the business of (1), and many undertake to do (2).

In the first section of the paper, I will argue that making the distinction between the contexts of pursuit and of justification opens a space to make progress in these conflicts between epistemic realism and constructivism. However, the discussion raises the issue of whether the contexts in fact are distinct, or whether justification collapses into pursuit. In the second section, I argue that employing a robust account of scientific problem-solving allows for a principled distinction between the contexts of pursuit and justification of scientific theories, based on weighing the theory's evidential support. In the final section, I elaborate on how weighing the evidence can contribute to eventual rational decision making in theory building.

\section{Pursuit and justification}

In his (1993), Allan Franklin distinguishes between the context of pursuit, in which a scientist or scientific community considers a theory to be worthy of further investigation, and the context of justification, in which theories are evaluated on the basis of

\footnotetext{
1 Massachusetts Institute of Technology (2009). As the article mentions, Planck himself anticipated this exception, but could not prove it existed.
} 
whether they are borne out by the evidence (p. $123 \mathrm{ff}$.). ${ }^{2}$ The distinction is particularly apt in the case I am examining, of novel results not predicted or explained by accepted theory. In the absence of a clear determination of whether the new evidence supports accepted theory or not, the scientific community must decide how to investigate the problems they wish to solve. In such a case, the decision of which theory or hypothesis to pursue is not determined univocally by the evidence. Nonetheless, once the theory has been pursued and more evidence gathered, it is possible to decide whether a given theory is supported by the evidence.

Franklin considers the history of the Fifth Force, a proposed modification of Newton's law of gravity to add a short-range force. The hypothesis of a fifth force was explored by Fischbach, Aronson, and others in response to the results of early experiments conducted by Eötvös, Pekar, and Fekete, among others. ${ }^{3}$ These early results were followed by those of Thieberger, Eöt-Wash, and Boynton (Franklin 1993, 108). The experimental results suggested that the Fifth Force hypothesis was worth pursuing - though to some scientists, it was worth pursing precisely to find evidence to refute it.

Identifying a distinct context of pursuit allows for a broader role for experiment than testing proposed hypotheses alone. Experiments often reveal phenomena of interest beyond testing the given hypothesis-they have a life of their own, as Hacking put it (1983). For instance, as Franklin points out, experiments related to the Fifth Force hypothesis are still going on, even though the hypothesis has been refuted-because the experiments themselves are of interest independently (p. 123).

My focus is on the case in which the scientific community is faced with experimental results that appear to be in conflict with accepted theory. In that case, scientists must decide how to proceed. As I argued above, this is the hard case for epistemic realists and constructivists alike. Scientists are faced with a problem that has not yet been solved, and they must decide how to go about investigating possible solutions. The situation raises the paradox of inquiry that Socrates poses in the Meno, 80d-e, and which Nickles (1981) paraphrases:

Either you know what you are searching for or you do not. If you do know, you already have it, whence inquiry is pointless. And if you do not know, you would not recognize it even if you stumbled on it accidentally; hence, again, inquiry is impossible, pointless. The way out of the paradox is to show that the second statement is false. You can know what you are looking for without already having it; you can know it without knowing it completely (p. 89).

In the case of nonconforming new results, a scientist can know that solving a given subproblem would contribute to a solution. Or, if she could establish conclusively that the experimental results are invalid, then the problem would no longer exist. Or, he could analyze the problem and discover which elements of it are amenable to inquiry. For instance, in the case of exceptions to Planck's law, no doubt research will be done

\footnotetext{
2 I would like to thank an anonymous reviewer for suggesting this distinction as a productive one for evaluating the debate under consideration, and for the references to the Franklin (1993) and Nickles (1980, and 1981) texts here.

3 Franklin (1993), 16ff., Fischbach et al. (1986), Eötvös et al. (1922).
} 
on the scope of the exceptions and the limitations of the law that must be built in to the theory, if the experiments are valid.

The process of pursuit is lawless, though it is constrained by the parameters of the problem. Choosing which subproblems to solve is a matter of making a decisiona decision that, in the case under consideration, is not fully rationally determined. Nickles observes that this has led the "friends of discovery," including himself, to conclude that "acceptance collapses into pursuit" entirely, that is, to deny the pursuitjustification distinction (p. 90). One way to so argue is equivalent to the constructivist position I described above. On such a view, scientists decide to pursue a theory because it is in keeping with their values, whether epistemic or social, or because it will get them grant money, or because it will enhance their standing in the community. According to this view, there is no independent way of weighing whether the theory pursued is supported by the evidence or is true. Another tack, which Nickles takes himself, is to argue that inquiry, interpreted as heuristic investigation into solutions to problems, is the primary activity of science. There is much that is advantageous about that standpoint.

But the pursuit-justification distinction in some form is valuable, and it is borne out by scientific practice. In fact, I will argue that Nickles's own account allows for a rigorous distinction in the context under investigation here, though perhaps not one that would be accepted by hard-core friends of justification. The question of whether a new or revised theory is worth pursuing can be detached from the question of whether, once the theory has been pursued over time, it solves a problem of interest to science. If evaluating whether a theory is justified comes down to evaluating its evidential support, then Nickles's and Franklin's accounts support a rigorous pursuit-justification distinction in the case under consideration. In what follows, I will show how pursuit and justification come apart in a particular case, that of Hermann von Helmholtz's construction of a theory of fluid dynamics. Then I will consider an objection, from Nickles himself: that not all scientific problems can be solved by evaluating evidential support.

\section{Reasons for pursuit: solving the problem of anomalous observations}

Hermann von Helmholtz, in the 19th century, noticed that several experimentally verified phenomena appeared to share a common set of observed regular motions. These phenomena included smoke rings, the motion of air around the ends of organ pipes, the wake of objects dragged through the water, and electromagnetic induced currents. Helmholtz himself was able to produce many of these effects in his lab. But he noticed that friction altered the regularity of the motions he was trying to capture.

At the time, there were two theories available to evaluate the motions, Euler's and Lagrange's. But neither theory could account for friction, nor could they solve the further problems for describing wave motion that Helmholtz was investigating. In 1858 , Helmholtz built a new theory of the motion of non-viscous fluids, incorporating three new laws of fluid motion. ${ }^{4}$ Helmholtz uses these laws to prove all the theorems

\footnotetext{
${ }^{4}$ Fuhs \& Shetz (1999). “(1) Fluid particles originally free of vorticity remain free of vorticity. (2) Fluid particles on a vortex line remain on a vortex line, so that vortex lines move with the fluid. (3) The strength of the vorticity is proportional to the length of the vortex line" (p. 736). Laws are restated in modern form.
} 
of the old theories, plus new theorems that reveal stable, invariant elements of fluid motion. Moreover, Helmholtz's equations and laws apply to a whole class of "fluids," including air, which allows him to extend his results to acoustics. (cf. Darrigol 2005, 155ff.)

There were several well-defined reasons why Helmholtz thought his theory worthy of pursuit. Helmholtz took up the problems of hydrodynamics and aerodynamics in the first place because of his interest in applying Green's theorems to solve problems in acoustics (Koenigsberger 1906, 167). Once he had constructed the theory of fluid motion, he found that the laws and descriptions could be extended to other types of wave motion, which was useful in building his theory of electromagnetism. Further, Helmholtz's goal was to build a theory that allowed him to show that measurable friction in fluids is the effect of the interaction between certain fluids (and waves of other kinds, including smoke rings and sound waves) in rotational motion. If Helmholtz's theory were to succeed, it would show that a model of the interaction between fluids explains the friction effects under investigation. In fact, Helmholtz was able to do so, but there was no guarantee, at the outset, that it would be possible. Reasons for pursuit were substantive, then: the theory was a promising way to bring together several related areas of scientific research, and it had the potential to solve significant scientific problems.

Once Helmholtz had constructed the theory, which appears to have taken him about two years, and once it had been tested experimentally over time, there were several reasons to consider it the best supported by the evidence. First, the fact that Helmholtz's theory can model friction quantitatively as arising from the interaction of fluids in motion, whereas Lagrange's and Euler's theories must treat it as an observed constant, is a powerful reason to accept Helmholtz's new theory. Helmholtz's account is an excellent example of a theory that can account for all of the old experimental results-he can derive all the theorems of Lagrange's and Euler's theories-as well as giving a novel explanation, of friction. The older physics had referred to substances, energy, heat, and so on, as indestructible elements shifting from place to place. By building models of regularities discovered and produced in experiment, Helmholtz explained heat in terms of friction and the contact of inelastic bodies, and energy in terms of the conservation of force exchanged in an interaction, in the same way that he had shown that friction in fluids is the result of interaction between rotating fluids.

This kind of conclusion was very effective over the 19th and early 20th centuries. Helmholtz's formulations were scientifically fruitful. They prompted theorems in mathematical physics by Clebsch, Riemann, and Hankel, had a profound influence on Kirchoff's classic lectures on mechanics, and Tait's work in knot theory, all of which had profound ramifications for physical theory, as well as Thomson's theory of vortex-ring atoms, which did not, because it was wrong (again, see Darrigol 2005, $155 \mathrm{ff})$. His work also is the origin of much recent work in quantum hydrodynamics including superfluids, especially Bose-Einstein condensates.

The method of problem solving employed here can be appreciated easily for its usefulness to science. Until then, friction had been "white noise," that is, a phenomenon that interfered with precise measurements and could not be quantified or explained by the theory. Once he had built his theory, Helmholtz was able to measure friction precisely, by showing how it could be modeled as an interaction. Euler and Lagrange had 
theories that accounted for fluid motion and proved certain theorems, but they could not account quantitatively for friction effects. They were good enough scientists not to try, so they included friction as a measurable but unanalyzed constant-as white noise. Helmholtz's theory allows an explanation of friction effects as interactions between waves.

Other examples of problem solving by explaining white noise are Rayleigh's and Ramsey's proof of the existence of argon, and Karl Jansky's proof that astronomical bodies were producing interference on a radio telephone line. ${ }^{5}$ Usually, these are described as Rayleigh's and Ramsey's discovery of argon and Jansky's discovery of cosmic radio waves. Certainly, Rayleigh, Ramsey, and Jansky discovered effects that were not explained by existing theory. But after having investigated and analyzed these effects over time, they were able at least to set the stage for solving the problems posed by the gap between observed phenomena and theory. Rayleigh and Ramsey demonstrated that argon gas is distinguishable from the other elements of the atmosphere by showing that its existence would explain anomalous atmospheric measurements, and Jansky showed that observed interference on a radio telephone line was consistent with what would be produced by astronomical bodies. Their proposals for solutions to problems are no less valuable to science than their discoveries.

Franklin's, Laudan's, and Nickles's pursuit-justification distinction bears up well in this context. Helmholtz, Rayleigh, Ramsey, and Jansky had reason to pursue their hypotheses about friction, argon, and radio waves initially, namely, the anomalous measurements. However, that reason was not a justification for the hypotheses themselves until after a lengthy process of investigating the hypotheses, their logical and substantive consequences, and the evidence for and against them. After research and investigation over time, pursuing those theories led to the solution of problems about the evidential support for the theory, i.e., about its justification.

Within the problem-solving model of inquiry, we go about investigating the justification of a theory in the following way. If we wish to establish whether a Fifth Force exists (or whether argon or cosmic radio waves exist), we

1. Pose the problem: Does a Fifth Force exist?

2. Pursue the hypothesis of the existence of a Fifth Force, and try to establish the parameters of the problem to be solved.

3. Pursue the theory, which will involve solving corollaries:

a. Were the initial experiments correct, or were there mistakes?

b. Has pursuit uncovered new results, consistent with or in conflict with the original theory?

c. What are the logical and substantive consequences of different theoretical configurations attempting to take these results into account?

d. Do some of these yield novel ways to test and to measure the theory's evidential support?

4. Finally, ask: Does one of the theories that survives the process of pursuit over time solve the initial problem?

5 Cf. Spanos 2009; Ghigo 2009. 
A theory can survive the above process, and can be established to be the best supported by the evidence, without proof that all the component claims of the theory are true in the realist sense. Helmholtz's theory is a good example, since it incorporates the idealizing assumption that all fluids are perfect continua, which we know to be untrue (cf. Smith 1998, 253ff.). This leads Nickles to argue that the pursuit-justification distinction collapses entirely.

But Nickles himself allows for a limited type of justification that is independent of reasons for pursuit: acceptance by the scientific community that a solution to a problem exists. This is, by definition, distinct from recognition that there is a reason to pursue the theory in order to solve that problem. Nickles sums up his observations of the scientific community's decisions as follows:

1. Many scientific problems are ill structured, and for substantive ( $v s$. purely formal, methodological) reasons. E.g., there exists no algorithm for determining whether something is a solution.

2. Despite 2, scientists make reliable judgments about when a problem has been solved. There frequently is near unanimity of agreement.

3. Scientists can sometimes make reliable judgments about the solvability or unsolvability of still unsolved problems, including solvability in terms of a given body of theory and the amount of time and effort required to obtain a solution-and thus evaluate the likely success of alternative research proposals and programs (Nickles 1981, 87).

Nickles continues to ask, "How is all this possible?" The simplest way to explain the reliability and unanimity of scientists' judgments, perhaps, is to appeal to the evidential support for a given theory. Nickles argues against this view, though, because to him problem-solving in science is not limited to determining whether a theory is best supported by the evidence.

Nickles contends that empiricist models of problem-solving do not account well for solutions to conceptual, "deep" problems that have no phenomenological component (Nickles 1981, 90ff). For Nickles, establishing whether a theory is best supported by the evidence solves only some scientific problems, and not necessarily the most interesting ones. Of course, Nickles's point is especially pressing in the case of string theory. Evaluating string theory merely on the basis of its evidential support would not solve the deeper problems of its possible fruitfulness (mathematical and physical) and unifying power.

But string theory falls outside the case I examine, the case in which new experimental results challenge accepted theory. The motivation for the construction of string theory is partly the promise of mathematical and physical fruitfulness, and partly the promise of a unified theory of gravitation. In contrast, in the cases I'm examining, the configuration of the problem itself requires that the evidence be weighed. Arguments that the accepted theory is mathematically fruitful or solves a deep conceptual problem might be revealing and productive, but do not solve the problem at issue here, of how to deal with the results in conflict with that theory.

Nickles identifies a profoundly interesting class of conceptually "deep" problems. The existence of such problems certainly shows why it would be worthwhile to pursue theories, such as string theory, with little or no evidential support, or which make few 
empirical predictions, or even none. But Nickles's arguments against empiricism aren't intended for the context under examination, in which scientists must explain nonconforming experimental results. The initial problem is a conflict between evidence and theory. The solution to the problem must account for that conflict, whether by showing that the evidence is invalid, by revising the existing theory, or by constructing a new theory not in conflict with the evidence. That construction may involve solving deep logical problems, what Laudan calls internal problems, about how to reconfigure the theory. But ultimately, evaluating whether the problem is solved must involve weighing the theory's evidential support.

\section{Theories, problem-solving, and justification}

I have argued that the context of pursuit, of scientific investigation, is distinct in at least some cases from the context of justification of particular scientific claims or theories. Now, the key question arises: is there a way to show that, in the cases under consideration, weighing the evidence can show that a given theory is justified? Or is it the case that the only criterion for acceptance of scientific theories is whether they are worth pursuing? I will argue for the former, in the cases under consideration.

To see why this is the case, we will return to the initial question: what should the scientific community do when presented with experimental results that seem to contradict accepted theory? Here, I will give an alternative answer to Nickles's question about how the reliability and unanimity of the scientific community's solutions to problems is possible. There are three general ways to adjust to the new results:

(1) You can investigate the experimental results more closely, and, over time, you may uncover further results that support or falsify the original results;

(2) You can decide to pursue a revised version of the current theory, and can investigate further, over time, whether the revised theory can account for the experimental results;

(3) You can decide to pursue an entirely new theory.

In the context under investigation here, there is no fully rationally determined way to choose between these approaches at first. In the context of pursuit, all three approaches might appear equally warranted. There may be no way to tell, initially, whether a scientist should pursue a revised theory or an entirely new theory, or should question and check the experimental results.

The lack of a rational way to decide initially between the three options does not imply that there never will be rational grounds on which to decide, though. While the constructivist account gives a good reading of the first steps in reacting to nonconforming results, one can argue nonetheless that the end stages of inquiry can be rationally or at least objectively driven (even if, in practice, they are not in some cases).

First, a constructivist might argue that in the case of choice (1), there is no principled way to decide whether a given experiment is valid or not. It is true that by adding ceteris paribus clauses or auxiliaries, it is possible to derive almost any result. But it is not true that you always can do so while avoiding inconsistency or preserving the integrity or potency of the theory. If you revise the theory in such a way that it is now not possible to derive some or any of the theory's original results, that is a powerful 
reason-an epistemic reason-to choose not to do so. There may be other reasons to argue that the new theory need not conserve those particular results. As Kuhn observes, Newton did not conserve Aristotle's analysis of change and motion. ${ }^{6}$ However, if a proposed new theory does not conserve any law of gravitation, for instance, there had better be an excellent argument for why it need not do so for it to be accepted.

Further, there is a fact of the matter about whether Collins's experimenter's regress is possible in a given case. Many if not most scientific experiments employ known principles, instruments, assumptions, and techniques that are used in a wide range of other theories, perhaps in entirely different disciplines. For instance, chemical spectrum analysis is used in many disciplines, including forensics, chemistry, and biology. It is not as easy as one might think simply to recalibrate an instrument or to reform a technique to get a more conformable result, especially if that would require you to calibrate it differently than thousands of other scientists do every day, and then to explain to them why you did so at the conference where you present your work. Franklin points out that one epistemic use of experiment simply is to recheck and to calibrate one's instruments to reproduce known and accepted results:

Other strategies are needed to validate the observation. These may include:

(1) Experimental checks and calibration, in which the experimental apparatus reproduces known phenomena. For example, if we wish to argue that the spectrum of a substance obtained with a new type of spectrometer is correct, we might check that this new spectrometer could reproduce the known Balmer series in hydrogen. If we correctly observe the Balmer Series then we strengthen our belief that the spectrometer is working properly. [...]

(2) Reproducing artifacts that are known in advance to be present (Franklin 2009, Sect. 1.1.1).

These reasons apply, mutatis mutandis, to weighing whether option (2) is possible, given all the available evidence. In some cases, you can't revise the old theory in the light of the new evidence without rendering it inconsistent or ineffective, or you can't explain away the experimental results without rendering invalid a raft of known experimental results (assuming there are good reasons to avoid this in the given case). In this case, constructing a new theory will look like a good option.

In the case of option (3), some constructivists emphasize the fact that more than one theory may be justified by the evidence. In some or even many cases, a single new theory cannot be shown to be entailed uniquely by the evidence or its consequences. I don't contest that. But that is not relevant to the question of whether the decision to construct a new theory can be made rationally, by evaluating experimental results and conceptual resources obtained over time, through the process of pursuit. It can be the case that more than one new theory is supported by the evidence, while remaining true that the old theory cannot be revised to account for the new results as effectively.

Given all this, the fact that the scientific community is in remarkable agreement on many cases is not surprising. It would be surprising if scientists were presented with a group of propositions or hypotheses and were told to decide right away whether those propositions were true, and if, under those circumstances, they agreed reliably with

6 Cf. Kuhn 1987, 7ff. 
each other. But given that scientists who are working on the same problem usually are working with similar instruments and conceptual repertoire, it is reasonable that inquiry over time will reveal a broad basis for agreement on how to resolve a lack of fit between evidence and theory. ${ }^{7}$

In this account, the constructivist is right that the scientific community is involved at certain levels of decision-making. For instance, it may have to be decided through discussion and dialogue whether existing experimental results should be abandoned, revised, or conserved in light of the novel results, given the state of knowledge at a given time. Good sense is useful here, as is diplomacy. The epistemic realist is correct, against the constructivist, that the decision to accept a given theory as justified ultimately can be made on rational and empirical grounds, in the cases under discussion. The epistemic realist is incorrect insofar as she argues that these grounds have to do exclusively with the truth of specific scientific claims. Belief in the truth of particular claims, however justified it may be at the end stages of inquiry, is not the most profound basis of the remarkable agreement of the scientific community on solutions to a given problem. Evidential and logical constraints revealed over time, through the process of inquiry and theory construction, provide a broad basis for the harmony of scientists' decisions.

The existence of new evidence in conflict with an accepted theory is not merely an engine of linear change from one theory to the next. It provokes a broad set of questions, which lead the scientific community to make decisions about which theories or hypotheses to pursue, to aid in solving the problem of nonconforming evidence. These decisions about what to pursue are risky - they are not determined fully by the available evidence. But ideally, the evidence gained in scientific pursuit is weighed broad-mindedly; it is not evaluated only in terms of whether it supports theory A or theory B. Instead, evidence is taken as a set of clues to the parameters of the problems to be solved-as solutions to the paradox of inquiry. Ideally, the process of pursuit will yield results that indicate the kinds of solutions to the problem that are available, and may even pose new problems, the solutions to which promise new solutions to the initial problem. Thus, with luck, rigorous further pursuit of a theory or hypothesis will lead to a narrowing down of the available options for theory building, and to proposed solutions to the problems identified as worthy of pursuing.

Once the options for theory building have been narrowed and solutions proposed, the question of the justification of the theory comes into play. Here, it may be the case that certain elements of the theory still are considered not fully justified, and are worthy of further pursuit-Helmholtz's continuity assumption, for instance. Nonetheless, it is reasonable to say that a theory is justified if it solves the initial problem that provoked pursuit of the theory, and does so better than at least most of its rivals. Using the problem-solving model of inquiry, then, it is possible to construct an evidence-based

\footnotetext{
7 The converse is also true: differing equipment or conceptual resources can threaten that agreement. When Heinrich Hertz first publicized his experiments in electromagnetic wave propagation, his results were challenged almost instantly by Sarrasin and de la Rive in France, who recreated Hertz's experiment and did not get the same result. It turned out that their equipment, including the room in which the experiment was done, differed from Hertz's, which explained the different results (cf. Poincaré 1890).
} 
view, that pursuit of a new or revised theory can lead eventually to solving the problem of nonconforming results.

Acknowledgments An anonymous reviewer made many invaluable suggestions for revision, which have been very effective and to the great benefit of the paper. I am grateful for comments received on a early version of this paper at a conference at Virginia Tech in spring 2009, from Bengt Autzen, Clark Glymour, Katrin Hohl, Deborah Mayo, and Aris Spanos. A revised version of the paper was presented to a reading group at the Center for Philosophy of Science at the University of Pittsburgh in fall 2009, at which there was a very valuable discussion, which included contributions from Lisa Damm, Natalie Gold, Michela Massimi, John Norton, Slobodan Perovic, Athanassios Raftopoulos, and Mark Sprevak.

\section{References}

Collins, H. (1994). A strong confirmation of the experimenter's regress. Studies in History and Philosophy of Modern Physics, 25(3), 493-503.

Darrigol, O. (2005). Worlds of flow: A history of hydrodynamics from the Bernoullis to Prandtl. Oxford: Oxford University Press.

Eötvös, R., Pekar, D., \& Fekete, E. (1922). Beiträge zum Gesetze der Proportionalität von Trägheit und Gravität. Annalen der Physik, 68, 11-66.

Fischbach, E., Sudarsky, D., Szafer, A., Talmadge, C., \& Aronson, S. (1986). Reanalysis of the Eötvös experiment. Physics Review Letters, 56, 3-6.

Franklin, A. (1986). The neglect of experiment. Cambridge: Cambridge University Press.

Franklin, A. (1993). The rise and fall of the fifth force: Discovery, pursuit, and justification in modern physics. New York: American Institute of Physics.

Franklin, A. (2009). Experiment in physics. The stanford encyclopedia of philosophy (Spring 2009 Edition), Zalta, E. N. (Ed.), URL = < http://plato.stanford.edu/archives/spr2009/entries/physics-experiment/ > .

Franklin-Hall, L. (2005). Exploratory experiments. Philosophy of Science, 72(5), 888-899.

Fuhs, A. E., \& Shetz, J. A. (Eds.). (1999). Fundamentals of fluid mechanics. New York: John Wiley.

Galison, P. (1987). How experiments end. Cambridge: Cambridge University Press.

Ghigo, F. (2009). Karl Jansky and the discovery of cosmic radio waves. National Radio Astronomy Observatory, http://www.nrao.edu/whatisra/hist_jansky.shtml.

Hacking, I. (1983). Representing and intervening: Introductory topics in the philosophy of natural science. Cambridge: Cambridge University Press.

Koenigsberger, L. (1906). Hermann von Helmholtz, translated by Frances A. Welby with a preface by Lord Kelvin. New York: Dover Publications.

Kuhn, T. (1987). What are scientific revolutions? In L. Kruger, L. Daston, \& M. Heidelberger (Eds.), The probablistic revolution, Volume I: Ideas in history (pp. 7-22). Cambridge, MA: MIT Press.

Laudan, L. (1981). A confutation of convergent realism. Philosophy of Science, 48(1), 19-49.

Massachusetts Institute of Technology. (July 31, 2009). Breakdown In Planck's Law: Bringing Objects Close Together Can Boost Radiation Heat Transfer. Science Daily: http://www.sciencedaily.com/ releases/2009/07/090730154025.htm.

Mayo, D. (1996). Error and the growth of experimental knowledge. Chicago: University of Chicago Press.

Nickles, T. (Ed.). (1980). Scientific discovery: Case studies. Dordrecht: D. Reidel.

Nickles, T. (1981). What is a problem that we may solve it? Synthese, 47, 85-118.

Poincaré, H. (1890). Letter to Heinrich Hertz, 8 October 1890. ALS 4p. HS 02996, Archiv, Deutsches Museum.

Smith, P. (1998). Approximate truth and dynamical theories. The British Journal for the Philosophy of Science, 49(2), 253-277.

Spanos, A. (2009). The discovery of argon: A case for learning from data? Philosophy of Science, 77, 359-380. 